\title{
Nonlinear dynamics analysis of tilting pad journal bearing-rotor system
}

\author{
Jiayang Ying*, Yinghou Jiao and Zhaobo Chen \\ School of Mechanical Electronic Engineering, Harbin Institute of Technology, Harbin, 150001, P.R. China
}

Received 4 February 2010

Revised 26 May 2010

\begin{abstract}
The nonlinear dynamics theory is increasingly applied in the dynamics analysis of tilting pad journal bearing-rotor system. However, extensive work on system dynamics done previously neglects the influence caused by the moment of inertia of the pad. In this paper, a comparison is made between the responses of the rotor in the bearings with and without pad inertia effect. Taking the Jeffcott rotor system as an example, the characteristics of bearing-rotor system, such as bifurcation diagram, cycle response, frequency spectrum, phase trajectories, and Poincaré maps, were attained within a certain rotation rate range. The pivotal oil-film force of tilting pad journal bearing was calculated by database method. The results directly demonstrate that considering the influence of the pad moment of inertia, system dynamics characteristics are found more complicated when rotor-bearing system works around natural frequency and system bifurcation is observed forward when rotor-bearing system works on high-speed range.
\end{abstract}

Keywords: Tilting pad journal bearing, nonlinear dynamics, pad moment of inertia

\section{Nomenclature}

$O_{r} \quad$ Geometrical center of the disk

$O_{m} \quad$ Barycenter of the disk

$O_{j} \quad$ Center of the shaft journal

$m \quad$ Static load

$k \quad$ Stiffness of the axial

$e_{u} \quad$ Selative imbalance modulus

$\omega \quad$ Shaft speed

$\omega_{k} \quad$ Natural frequency

$\phi_{e} \quad$ Initial phase angle $\left(\varphi_{e}=0\right)$

$F \quad$ Oil-film force

$x, y \quad$ Instantaneous displacement

$\ddot{x}, \ddot{y} \quad$ Acceleration

$\omega \quad$ Rotor speed

$g \quad$ Acceleration of gravity

$X, Y \quad$ Instantaneous displacement

$S \quad$ Dimensionless rotor speed ratio $\left(S=\omega / \omega_{k}\right)$

$E_{u} \quad$ Dimensionless eccentricity of disk

$G$ Dimensionless acceleration of gravity $\left(G=g / \omega^{2} c\right)$ 


$\begin{array}{ll}K & \text { Dimensionless stiffness of the axial } \\ F_{o x}, F_{o y} & \text { Dimensionless oil-film forces in Cartesian coordinates } \\ \delta_{i} & \text { Pivot position angle of a pad } \\ m_{i} & \text { Pre-load modulus } \\ \psi_{\text {min }} & \text { Minimum clearance radio of the bearing } \\ \psi_{v i}=\psi_{i} / \psi_{\text {min }} & \text { Relatively clearance radio of a pad } \\ \alpha_{i}, \dot{\alpha}_{i} & \text { Angular displacement and the swing palstance of a pad } \\ \ddot{\alpha}_{i} & \text { Angular acceleration of a pad } \\ I & \text { Moment of inertia of a pad } \\ f_{t i} & \text { Oil-film force components in the tangential directions } \\ s & \text { Thickness of pad }\end{array}$

\title{
Subscripts
}

\author{
$i=1,2,3 \quad$ Number of pad \\ $r, j \quad$ Refer to the disk and the journal
}

\section{Introduction}

Tilting pad journal bearings (TPJB) are commonly used for supporting the rotors of high speed rotating machines, such as turbines or generators. Tilting pad journal bearings have many advantages in particular their inherent stability and self-alignment ability. The degree of freedom increases because of the swing of the pad, which makes dynamics analysis of tilting pad journal bearing-rotor system more difficult than that of fixing pad [1-3]. Considering the non-linear factors appearing in actual rotor systems, such as oil-film force and sealing force, non-linear theory is applied to replace inaccurate linear dynamics theory. Until now the moment of inertia of a pad is always neglected in most results which are based on nonlinear analysis.

Extensive work has been done to understand the dynamics characteristics of bearing and bearing-rotor system. Gadangi and Palazzolo [4] concluded that pad deformations greatly increase the amplitude of the journal center's orbit. Monmousseau and Fillon [5] stated that the dynamic response of the bearing becomes the maximum at the critical frequency. Adams and Payandeh [6] found that the behavior of statically unloaded pads is a strong sub-synchronous self-excited vibration. Dimond, Sheth, Allaire and He [7] concluded that removing the additional dynamics from the traditional contact excitation methods is an advanced way to reduce measurement uncertainty. Feng and Chu presented in [8], a study about the influence of preload in tilting-pad bearing on lateral vibrations of a vertical assembly, concluding that when the preload is applied to more than one pad the influence of this parameter is reflected not just in the vibration amplitude, but also in the shaft orbit inside the bearing. Michalski, Zindeluk and Rocha [9] verified the influence of journal bearing axial grooves on the dynamic behavior of horizontal rotors comparing the results between a simple plain bearing and the others semi-circular axial grooved journal bearings. Tiwari and Friswell [10] presented an extensive survey of a wide variety of bearing and seal identification tests for dynamic coefficients. Hashimoto et al. [11] analyzed the dynamic characteristics of a cylindrical turbulent journal bearing by the use of the short bearing theory with the Gümbel's boundary condition, and they demonstrated that turbulent effects became more significant as the rotor speed increased. Brockwell and Dmochowski [12] used a more direct method of measuring the bearing dynamic properties of journal bearings by generating two distinct, straight line orbits of vibration. To obtain such orbits, the magnitude of two oscillating forces, as well as the phase difference between them, was carefully adjusted.

The objective of this work is to get dynamics characteristics of tilting pad journal bearing-rotor system considering the moment of inertia of a pad. The characteristics, such as bifurcation diagram, cycle response, frequency spectrum, phase trajectories, and Poincaré maps, were attained within a certain rotation rate range. The results depicted the alternation of the rotor system among the periodic, quasi-periodic and chaotic motions caused by the rotation speed changed. 


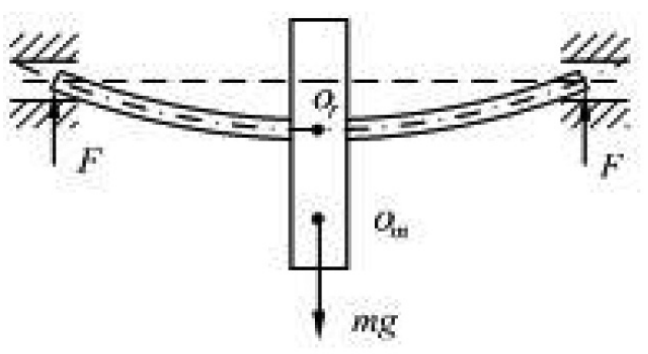

(a) Flexible Jeffcott model

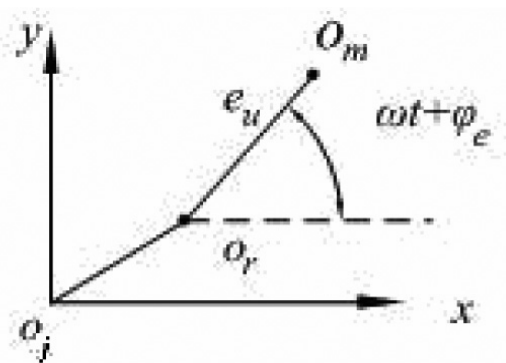

(b) Coordinate system

Fig. 1. Computer model and coordinate system.

\section{Dynamics model}

The first step of analyzing bearing-rotor system is mathematical modeling. Figure 1(a) shows the discussed model of the flexible Jeffcott rotor system. The rotor system is a symmetric flexible rotor with a central disk, where it is possible to change the unbalance mass. The journals in the ends of the rotor are the parts of the shaft in contacting with the oil film. The TPJBs supporting the rotor are three-pad TPJBs. As shown in Fig. 1(a), the lumped mass of shaft is omitted. Figure 1(b) shows the coordinate system attached to shaft center used in numerical integration.

The mathematical equations of the model shown in Fig. 1(a) can be described by the flowing differential equation.

$$
\left\{\begin{array}{l}
m \ddot{x}_{r}-k\left(x_{j}-x_{r}\right)=m e_{u} \omega^{2} \sin \left(\omega t+\varphi_{e}\right) \\
m \ddot{y}_{r}-k\left(y_{j}-y_{r}\right)=m e_{u} \omega^{2} \cos \left(\omega t+\varphi_{e}\right)-m g
\end{array}\right.
$$

After being nondimensionalized and transposed, the dynamics equations shown as Eq. (1) are transformed into Eq. (2).

$$
\left\{\begin{array}{l}
Z_{1}^{\prime}=Z_{3} \\
Z_{2}^{\prime}=Z_{4} \\
Z_{3}^{\prime}=-{ }_{S^{2}}^{1}\left(X_{r}-X_{j}\right)+E_{u} \sin (\omega t) \\
Z_{4}^{\prime}=-{ }_{S^{2}}^{1}\left(Y_{r}-Y_{j}\right)+E_{u} \cos (\omega t)-G
\end{array}\right.
$$

where $Z=\left[Z_{1}, Z_{2}, Z_{3}, Z_{4}\right]^{T}=\left[X_{i}, Y_{i}, \dot{X}_{i}, \dot{Y}_{i}\right]^{T}$.

At the journal position, the lumped mass of a shaft omitted in the dynamics model is requited in attaining the velocity of a shaft. The search method provides a way to solve the neglect of the lumped mass by considering the balance of a shaft. Equation (3) represents the search target, where $\gamma$ is a small number.

$$
\left\{\begin{array}{l}
\left|2 F_{o x}-K\left(X_{r}-X_{j}\right)\right| \leqslant \gamma \\
\left|2 F_{o y}-K\left(Y_{r}-Y_{j}\right)\right| \leqslant \gamma
\end{array}\right.
$$

\section{Nonlinear oil-film force}

Oil-film force is the most important part in bearing-rotor system. Reynolds equation is now considered as the most accurate way to obtain the oil-film force. However, solving Reynolds equation costs a lot of time. The database method [13] is an effective way to obtain the oil-film force. This method calculates Reynolds' Equation to establish oil-film forces database of a single pad, and then obtains the value by searching the database and joining up the component searched. The database is based on a pad angular extension and length to diameter ratio.

The key of nonlinear oil film force database method is to determine the scope of the parameter perturbation. The dynamic Reynolds equation [14] is:

$$
\partial \phi\left(\begin{array}{r}
H^{3} \partial P \\
\partial \phi
\end{array}\right)+\left(\begin{array}{c}
D \\
L
\end{array}\right)^{2} \begin{gathered}
\partial \lambda \\
\partial \lambda
\end{gathered}\left(\begin{array}{r}
H^{3} \partial P \\
\partial \lambda
\end{array}\right)=-6 \varepsilon\left(1-2 \theta^{\prime}\right) \sin \phi+12 \varepsilon^{\prime} \cos \phi
$$

Set $R S=-6 \varepsilon\left(1-2 \theta^{\prime}\right) \sin \phi+12 \varepsilon^{\prime} \cos \phi$, three conditions can be deduced as following: 


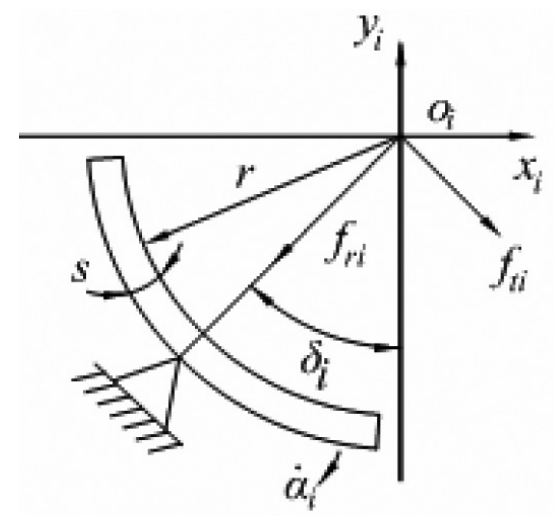

Fig. 2. Balance schematic diagram.

1) If $\varepsilon\left(1-2 \theta^{\prime}\right)=0, \varepsilon^{\prime}=0$, then $F_{x}=F_{y}=0$.

2) If $\varepsilon\left|1-2 \theta^{\prime}\right| \geqslant 2\left|\varepsilon^{\prime}\right|$, set $P_{1}=P /\left(\varepsilon\left|1-2 \theta^{\prime}\right|\right), q_{1}=2 \varepsilon^{\prime} /\left(\varepsilon\left|1-2 \theta^{\prime}\right|\right)$.

When $\varepsilon\left(1-2 \theta^{\prime}\right)>0, R S(1)=-6 \sin \phi+6 q_{1} \cos \phi$.

When $\varepsilon\left(1-2 \theta^{\prime}\right)<0, R S(2)=6 \sin \phi+6 q_{1} \cos \phi$.

3) If $\varepsilon\left|1-2 \theta^{\prime}\right| \leqslant 2\left|\varepsilon^{\prime}\right|$, set $P_{2}=P /\left(2\left|\varepsilon^{\prime}\right|\right), q_{2}=\varepsilon\left(1-2 \theta^{\prime}\right) /\left(2\left|\varepsilon^{\prime}\right|\right)$.

When $\varepsilon^{\prime}>0, R S(3)=-6 q_{2} \sin \phi+6 \cos \phi$.

When $\varepsilon^{\prime}<0, R S(4)=-6 q_{2} \sin \phi-6 \cos \phi$.

Through the way above, scopes of the parameter $\varepsilon^{\prime}$ and $\theta^{\prime}$ in Reynolds equation are changed to $q\left(q_{1}, q_{2}\right) \in(-1,1)$. Three parameters $\varepsilon \in(0,0.95), T H \in\left(0^{\circ}, 360^{\circ}\right)$ and $q\left(q_{1}, q_{2}\right) \in(-1,1)$ are used to build up the database of a single pad.

When obtaining the oil-film force, the position and velocity of the shaft are already known. $\varepsilon, \theta, \varepsilon^{\prime}$ and $\theta^{\prime}$ can be obtained by Eq. (4), also $q\left(q_{1}, q_{2}\right)$ can be got by the formula in the three conditions above.

$$
\left\{\begin{array}{l}
\theta^{\prime}=\dot{Y}_{j} \sin \theta-\dot{X}_{j} \cos \theta \\
\theta=\arctan X_{j} / Y_{j} \\
\varepsilon^{\prime}=-\dot{X}_{j} \sin \theta-\dot{Y}_{j} \cos \theta \\
\varepsilon=X_{j}^{2}+Y_{j}^{2}
\end{array}\right.
$$

The coordinate system in the database establishment is attached to the center of the bearing while the coordinate system in numerical integration is attached to the center of the journal. In order to make the two coordinate systems consistent, transformation equations are set up to convert the position and velocity of the journal. The $\left[X_{i}, Y_{i}, \dot{X}_{i}, \dot{Y}_{i}\right]^{T}$ obtained by Eq. (5) can be identified as the state variables used for obtaining the oil-film force.

$$
\left\{\begin{array}{l}
X_{i}=\left(X_{j}-A_{i} \cos \delta_{i}\right) / \psi_{v i}-m_{i} \sin \delta_{i} \\
Y_{i}=\left(Y_{j}+A_{i} \sin \delta_{i}\right) / \psi_{v i}-m_{i} \cos \delta_{i} \\
\dot{X}_{i}=\left(\dot{X}_{j}-\dot{A}_{i} \cos \delta_{i}\right) / \psi_{v i} \\
\dot{Y}_{i}=\left(\dot{Y}_{j}+\dot{A}_{i} \sin \delta_{i}\right) / \psi_{v i}
\end{array}\right.
$$

where $\dot{A}_{i}=\dot{\alpha}_{i} / \omega \psi_{\min }, A_{i}=\alpha_{i} / \psi_{\min }$.

Figure 2 is balance schematic diagram of a pad, which set up pendulum Eq. (6). The angular displacement of a pad is attained by integrating the swing palstance of a pad. The swing palstance of a pad is attained by integrating the angular acceleration of a pad. Considering the moment of inertia, the angular acceleration is calculated by Eq. (6). Calculation of the pendulum equation is simultaneous with dynamics equation, since the pad is regarded as one of the moving parts. Obviously the number of equations is increased. As a result, the time spent on numerical integration is longer, but the time devoted to obtain the pad's swing rate is saved. The results obtained by this method are more practical.

$$
I \ddot{\alpha}_{i}=f_{t i} s \quad(i=1,2 \cdots, n)
$$


Table 1

Specification of the system

$\begin{array}{lcc}\text { Parameter } & \text { Value } & \mathrm{Unit} \\ \text { Static load } & 1000 & \mathrm{~kg} \\ \text { Shaft stiffness } & 8.5873 \times 10^{7} & \mathrm{~N} / \mathrm{m} \\ \text { Eccentricity } & 8.55 \times 10^{-5} & \mathrm{~m} \\ \text { Pad angular extent } & 100 & \mathrm{deg} \\ \text { Radial clearance } & 2.85 \times 10^{-4} & \mathrm{~m} \\ \text { Mass moment of inertia of a pad } & 2.45 \times 10^{-4} & \mathrm{~kg} \cdot \mathrm{m}^{2} \\ \text { Rotor speed } & 150 \sim 1200 & \mathrm{rad} / \mathrm{s} \\ \text { Length to diameter ratio } & 0.5 & \\ \text { Preload modulus } & 0.6667 & \end{array}$

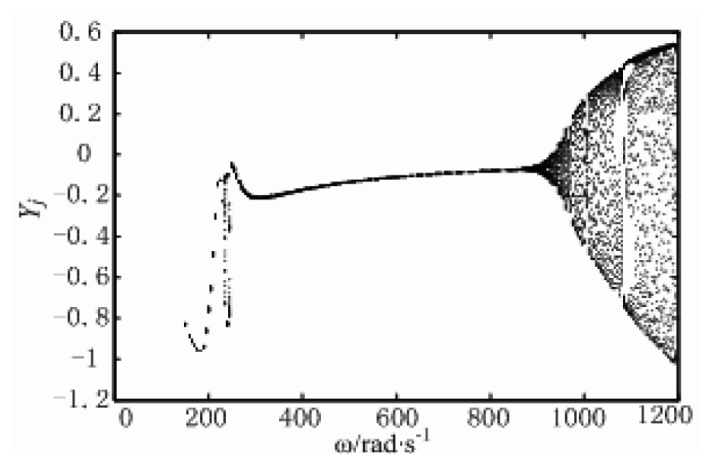

(a) Journal

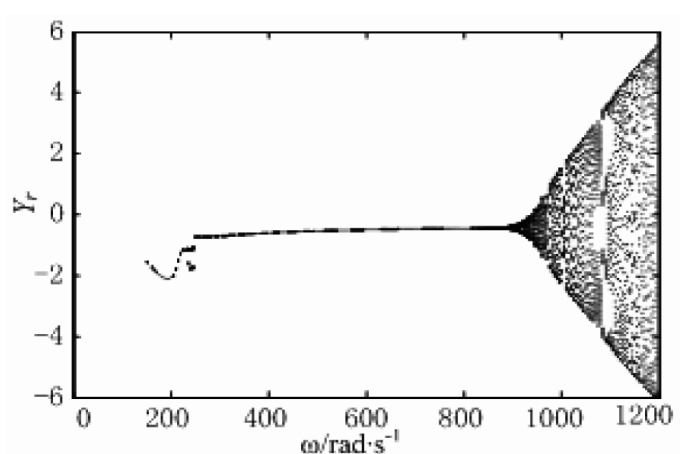

(b) Disc

Fig. 3. Bifurcation diagrams of the shaft and the disc (with the pad's moment of inertia).

\section{Numerical results and discussion}

The specification of the shaft and the tilting pad journal bearing with three pads is shown in Table 1. As the numerical integration takes long time, the range of rotor speed is set to $150 \sim 1200 \mathrm{rad} / \mathrm{s}$ for short.

Figure 3 shows the bifurcation diagram of the shaft and the disc at Y position taking in the inference of pad inertia. When the speed increases, it can be observed that periodic and quasi-periodic motion occurs in the range of 150 $<\omega<1200 \mathrm{rad} / \mathrm{s}$. When $150<\omega<230 \mathrm{rad} / \mathrm{s}$, the vibration of the system is small, the motion is synchronous, and the bifurcation jumps at this interval. When $230<\omega<250 \mathrm{rad} / \mathrm{s}$ the synchronous motion changes to subsynchronous motion with period-two for a short interval. When $250<\omega<880 \mathrm{rad} / \mathrm{s}$ the sub-synchronous changes to synchronous motion. At $880<\omega<920 \mathrm{rad} / \mathrm{s}$, the system response displays quasi-periodic motion, and the vibration of the system is also small. When $920<\omega<935 \mathrm{rad} / \mathrm{s}$ the quasi-periodic motion changes to sub-synchronous with period-seven for a short interval. At $\omega=935 \mathrm{rad} / \mathrm{s}$, the motion goes back to quasi-periodic. In Fig. 3, when 1080 $<\omega<1120 \mathrm{rad} / \mathrm{s}$, the bifurcation diagram depicts a sub-synchronous motion. However, the characteristics of the system at that range of speed demonstrate quasi-periodic motion. This misdescription of the bifurcation diagram is probably caused by the maximum time limitation of the numerical integration for each speed.

Figure 4 displays the bifurcation diagrams of rotor system at y axis path when ignoring pad Moment of inertia. The same in Fig. 3, when $\omega=200-300 \mathrm{rad} / \mathrm{s}$, the loop is observed. However when $\omega=235-245 \mathrm{rad} / \mathrm{s}$, system motion displays as synchronous motion, which is obvious in picture 3 . When $\omega=875 \mathrm{rad} / \mathrm{s}$, it enters almost periodic motion, the rotor speed is higher than in picture 3 . When $\omega>800 \mathrm{rad} / \mathrm{s}$, picture 3 and picture 4 both experience synchronous, quasi-periodic, sub-synchronous and quasi-periodic motion. From this comparison, the pad moment of inertia has no advance for the stability of system.

The characteristics of the system are displayed by the times-history, frequency spectrum, phase trajectories, and Poincare map in detail. Since the increasing process is similar to the decreasing process, the characteristic of the system is analyzed taking the case of decreasing process at the disc position as shown in figures followed.

In Fig. 5, where $\omega=240 \mathrm{rad} / \mathrm{s}$, neglecting the pad moment of inertia, synchronous behavior is inferred from the single point in Poincare map. The single period in time-history, the onefold curve in phase trajectories and the 


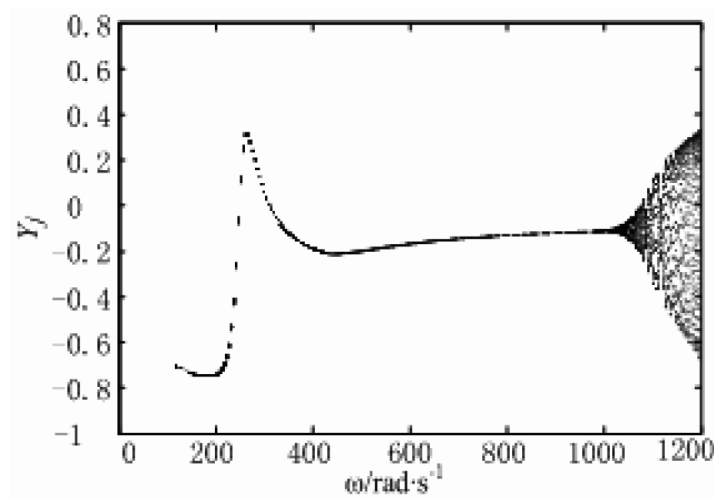

(a) Journal

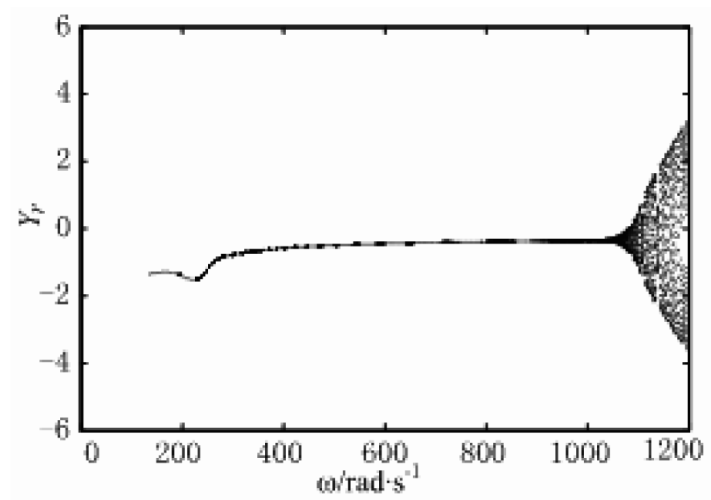

(b) Disc

Fig. 4. Bifurcation diagrams of the shaft and the disc (without the pad's moment of inertia).

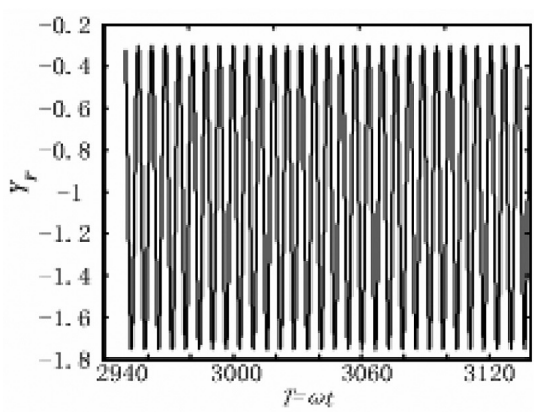

(a) Times-history

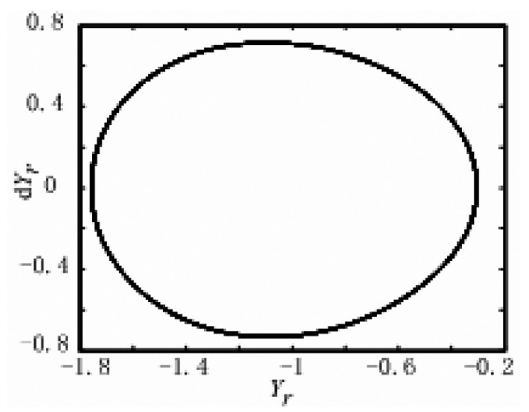

(c) Phase trajectories

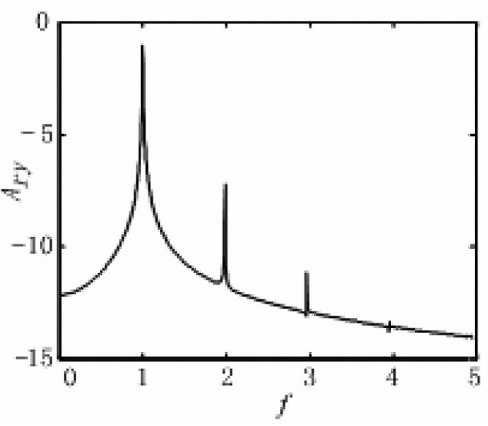

(b) Frequency spectrum

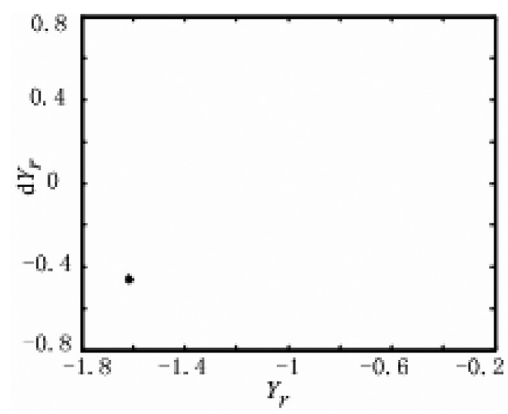

(d) Poincare map

Fig. 5. Characteristics of the system when $\omega=240 \mathrm{rad} / \mathrm{s}$ (without the pad moment of inertia).

frequency spectrum characterize synchronous motion. Figure 6 depicts dynamics characteristics considering the inference of pad moment of inertia when $\omega=240 \mathrm{rad} / \mathrm{s}$. Different with Fig. 5, there are two points in Poincare map, and two corves are observed in phase trajectories. Obviously, at this condition, system motion is sub-synchronous motion with period-two. This comparison confirms the results concluded from bifurcation diagrams.

\section{Conclusion}

Taking the influence of pad's moment of inertia into consideration, nonlinear dynamics characteristics are numerically calculated for the unbalanced rotor supported by a three-pad TPJB. The results obtained by method above 


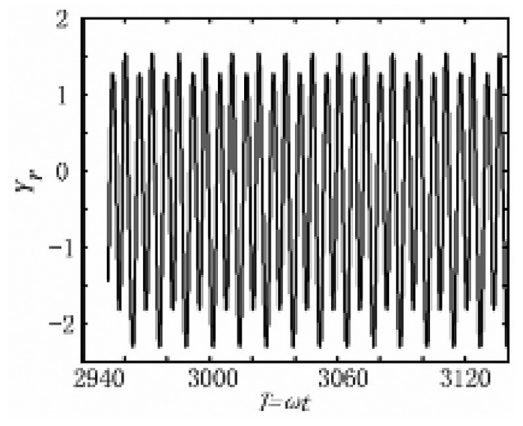

(a) Times-history

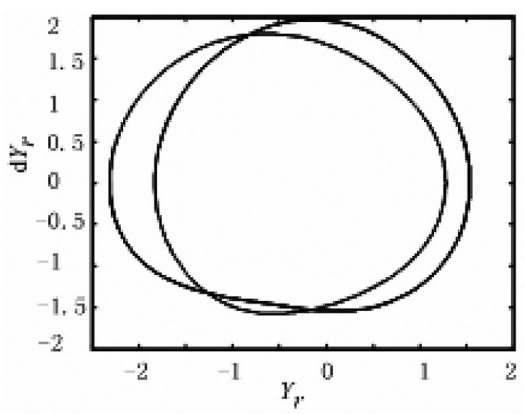

(c) Phase trajectories

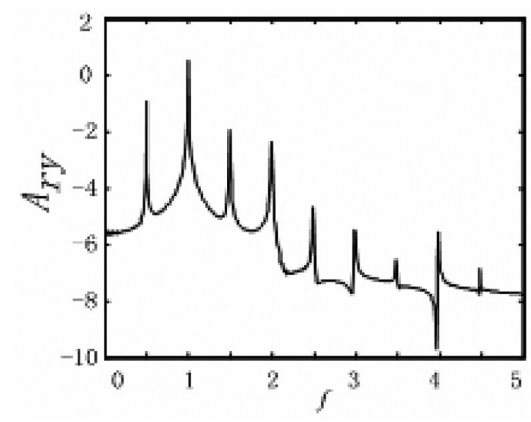

(b) Frequency spectrum

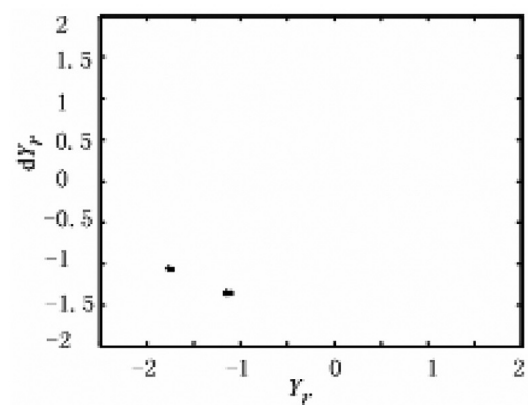

(d) Poincare map

Fig. 6. Characteristics of the system when $\omega=240 \mathrm{rad} / \mathrm{s}$ (with the pad moment of inertia).

are more practical, and the nonlinear dynamics analysis of tilting pad journal bearing-rotor system using database method is enriched.

Considering the influence of the pad moment of inertia, system dynamics characteristics are found more complicated when rotor-bearing system works around natural frequency and system bifurcation is observed forward when rotor-bearing system works on high-speed range. When rotor-bearing system works on other speed range, the pad moment of inertia has little effects on system dynamics characteristics. The results show that counting in pad moment of inertia has negative effects on system stability. In other words, when rotor-bearing system works on high-speed range and around natural frequency, pad moment of inertia should be considered to obtain more practical system characteristics.

Future investigations will focus on characterizing chaotic behavior of TPJB with more than three pads and analyzing dynamics characteristics of shafting supported by several TPJBs even with other kinds of bearings together.

\section{Acknowledgements}

Thanks to the supports of the National Nature Science Foundation of China (NSFC) (No. 10630240, 10872054 and 10872055). Thanks to the supports of the Heilongjiang province Nature Science Foundation of China (No. ZD200905).

\section{References}

[1] F. Chu and Y. Tang, Stability and Non-Linear Responses of A Rotor-Bearing System with Pedestal Looseness, Journal of Sound and Vibration 241(5) (2001), 879-893.

[2] Y.H. Jiao, Z.B. Chen, F.L. Liu, X.Q. Qu and Z.M. Zhang, Nonlinear Analysis of Unbalance Response For Jeffcott Rotor-tilting Pad Bearing System, Proceedings of the CSEE 12(24) (2004), 227-232.

[3] I. Abu-Mahfouz and M.L. Adams, Numerical Study of Some Nonlinear Dynamics of a Rotor Supported on a Three-Pad Tilting Pad Journal Bearing (TPJB), Journal of Vibration and Acoustics 127 (2005), 262-272. 
[4] R.K. Gadangi and A.B. Palazzolo, Transient Analysis of Tilt Pad Journal Bearings Including Effects of Pad Flexibility and Fluid Film Temperature, ASME J Tribol 117 (1995), 302-307.

[5] P. Monmousseau and M. Fillon, Frequency Effects on the TEHD Behavior of a Tilting-Pad Journal Bearing Under Dynamic Loading, ASME J Tribol 121 (1999), 321-326.

[6] M.L. Adams and S. Payandeh, Self-excited vibration of statically unloaded pads in tilting-pad journal bearings, ASME J Lubr Technol $\mathbf{1 0 5}$ (1983), 377-384.

[7] T.W. Dimond, P.N. Sheth, P.E. Allaire and M. He, Identification Methods and Test Results for Tilting Pad and Fixed Geometry Journal Bearing Dynamic Coefficients - A Review, Shock and Vibration 16 (2009), 13-43.

[8] F. Feng and F. Chu, Influence of pre-load coefficient of TPJBs on shaft lateral vibration, Tribol Int 35 (2002), 65-71.

[9] Miguel Angelo de Carvalho Michalski, Moys'es Zindeluk and Renato de Oliveira Rocha, Influence of Journal Bearing Axial Grooves on The Dynamic Behavior of Horizontal Rotors, Shock and Vibration 13 (2006), 285-300.

[10] R. Tiwari, A.W. Lees and M.I. Friswell, Identification of Dynamic Bearing Parameters: A Review, Shock and Vibrat Digest 36 (2004), 99-124.

[11] H. Hashimoto, S. Wada and J. Ito, An application of short bearing theory to dynamic characteristic problems on turbulent journal bearings, ASME J Tribol 109 (1987), 307-314.

[12] K.R. Brockwell and W. Dmochowski, Experimental Determination of the Journal Bearing Oil Film Coefficients by the Method of Selective Vibration Orbits, Proc. 12th Biennial ASME Conference on Mechanics Vibration and Noise 18 (1989), 251-259.

[13] W. Wang and Z.M. Zhang, Nonlinear oil-film force database, Journal of Shanghai University of Technology 14(4) (1993), 299-305, (in Chinese).

[14] R.G. Kirk and E.J. Gunter, Short Bearing Analysis Applied to Rotor Dynamics - Part I: Theory, Transactions of ASME, Journal of Lubricant Technology (January 1976).

[15] R.K. Gadangi and Palazzolo, Transient Analysis of Tilt Pad Journal Bearings Including Effects of Pad Flexibility and Fluid Film Temperature, ASME J Tribol 117 (1995), 302-307.

[16] E.P. Okabe and K.L. Cavalca, Rotordynamic analysis of systems with a non-linear model of tilting pad bearings including turbulence effects, Nonlinear Dyn 57 (2009), 481-495.

[17] R.G. Kirk, A. Alsaeed, J. Liptrap, C. Lindsey, D. Sutherland, B. Dillon, E. Saunders, M. Chappell, S. Nawshin, E. Christian, A. Ellis, B. Mondschein, J. Oliver and J. Sterling, Experimental Test Results for Vibration of a High Speed Diesel Engine Turbocharger, Tribology Transactions 51(4) (2008), 422-427.

[18] A. Muszynska, Whirl and Whip - Rotor/Bearing Stability Problems, Journal of Sound and Vibration 110(3) (1986), 443-462.

[19] R.G. Kirk, A.A. Alsaeed and E.J. Gunter, Stability Analysis of a High-Speed Automotive Turbocharger, Tribology Transactions 50(3) (2007), 427-434.

[20] B.C. Pettinato, R.D. Flack and L.E. Barrett, Effects of excitation frequency and orbit magnitude on the dynamic characteristics of a highly preloaded three-lobe journal bearing, Tribol Trans 44 (2001), 575-582.

[21] R.G. Kirk and Z. Guo, Influence of Leak Path Friction on Labyrinth Seal Inlet Swirl, Tribology Transactions 52(2) (2009), $139-145$. 

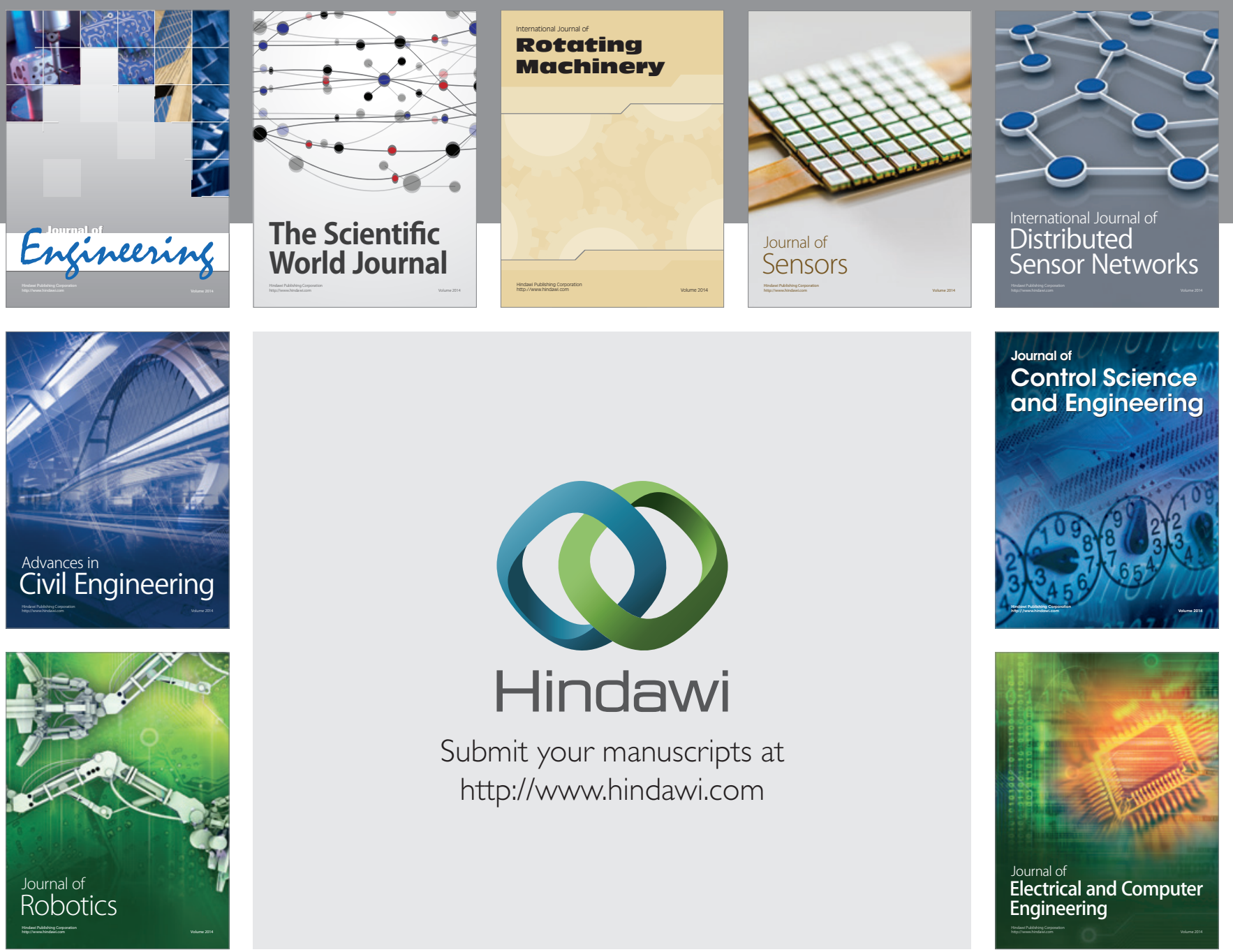

Submit your manuscripts at

http://www.hindawi.com
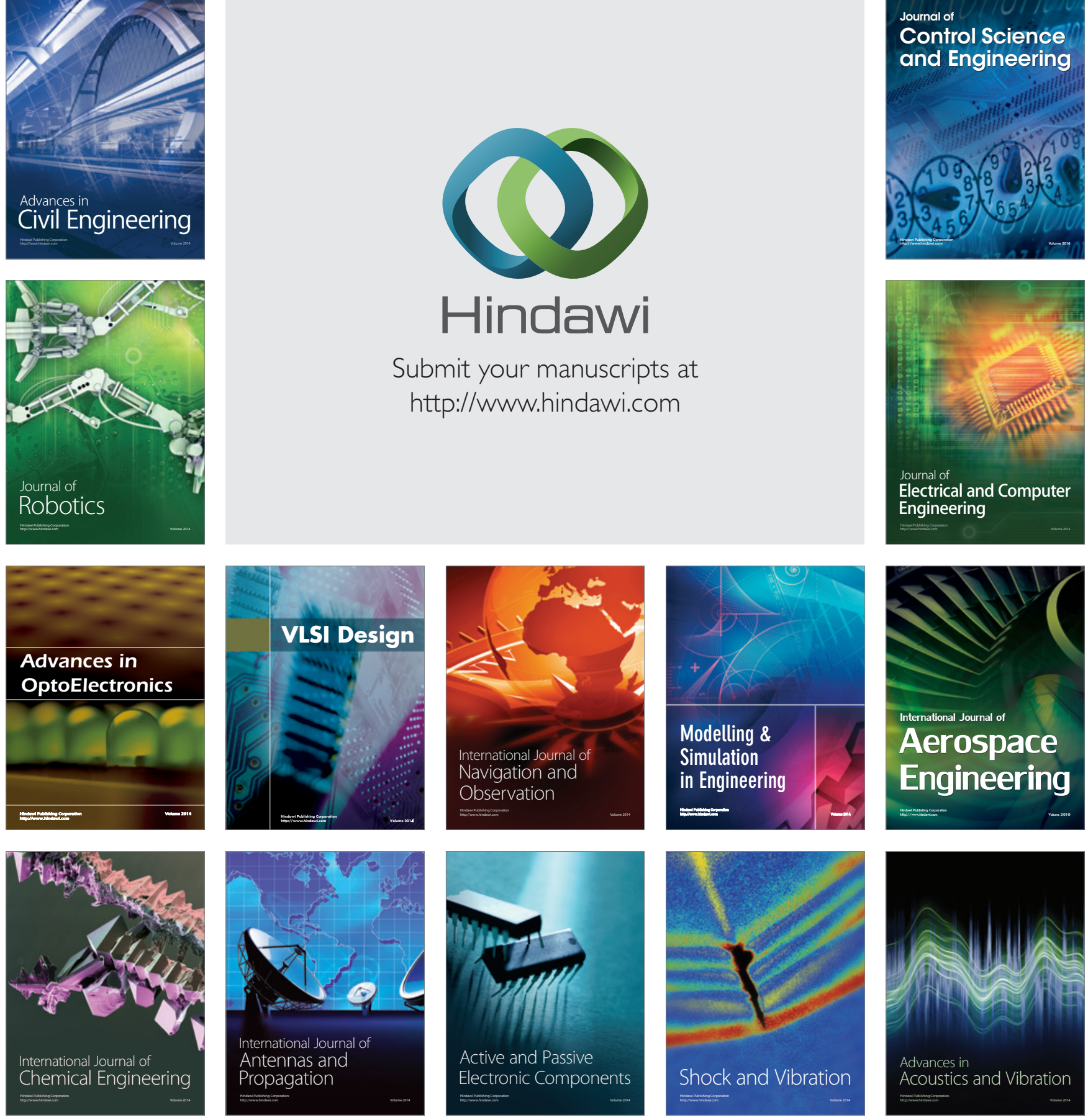